\title{
Electron Diffractive Imaging of a Single Nanoparticle
}

F.-R. Chen, ${ }^{* * *}$ R. Dronyak, ${ }^{* * *}$ C.-K. Feng, ${ }^{* *}$ K. S. Liang, ${ }^{*}$ and T.-K. Lee***

*National Synchrotron Radiation Research Center, Hsinchu 30076, Taiwan

**Department of Engineering and System Science, National Tsing Hua University, Hsinchu 30013, Taiwan

***Institute of Physics, Academia Sinica, Nankang, Taipei 11529, Taiwan

The electron diffraction pattern from a faceted $\mathrm{MgO}$ nanoparticle (see Fig. 1) was phased using coherent diffractive imaging (CDI) technique. The primary limitations of the accurate and reproducible inversion of the electron diffraction pattern are due to the difficulty in treating experimental noises, substrate scattering and partial spatial coherence of the incident beam. Furthermore, the reconstruction of the complex-valued exit wave function requires sufficiently tight boundary of the sample called

"support". In this work, we present the phase recovery procedure that utilizes guided hybrid input-output (GHIO) algorithm with dynamic support to reconstruct the stable and reproducible solution by optimizing feedback parameter of the algorithm and threshold value for dynamic support. To quantify the spatial accuracy of recovered result the deviation map was obtained as a relative standard deviation of the averaged solution. The result shows that the reproducible solution can be deduced from diffraction patterns only, without information from TEM image [1-2].

Figure 2 shows recovered shape and exit wave of the sample with their deviation maps. The contrast decay at the edges and corners of the sample is due to the thickness decrease of the faceted nanoparticle. The region of low accuracy in the exit wave is comparable with the resolution of the recovered shape function. It may be due to the large amount of missed weak scattering signals in the recorded diffraction patterns. Therefore, it complicates imaging the local structure at atomic resolution, such as for single-atom defects. The optimization analysis and effects of the incident illumination on the phase recovery technique will be discussed in detail.

\section{References}

[1] R. Dronyak, C.-K. Feng, K. S. Liang and F.-R. Chen. Electron diffractive imaging of the MgO nano-particle: towards atomic-resolution. XXI IUCr Congress and General Assembly, Osaka. Acta Cryst. A64, C130 (2008).

[2] This work was supported by the National Science Council (NSC 97-2112-M-213-001 and NSC 95-2221-E-007-093) and the Thematic Project of Academia Sinica of Taiwan. 


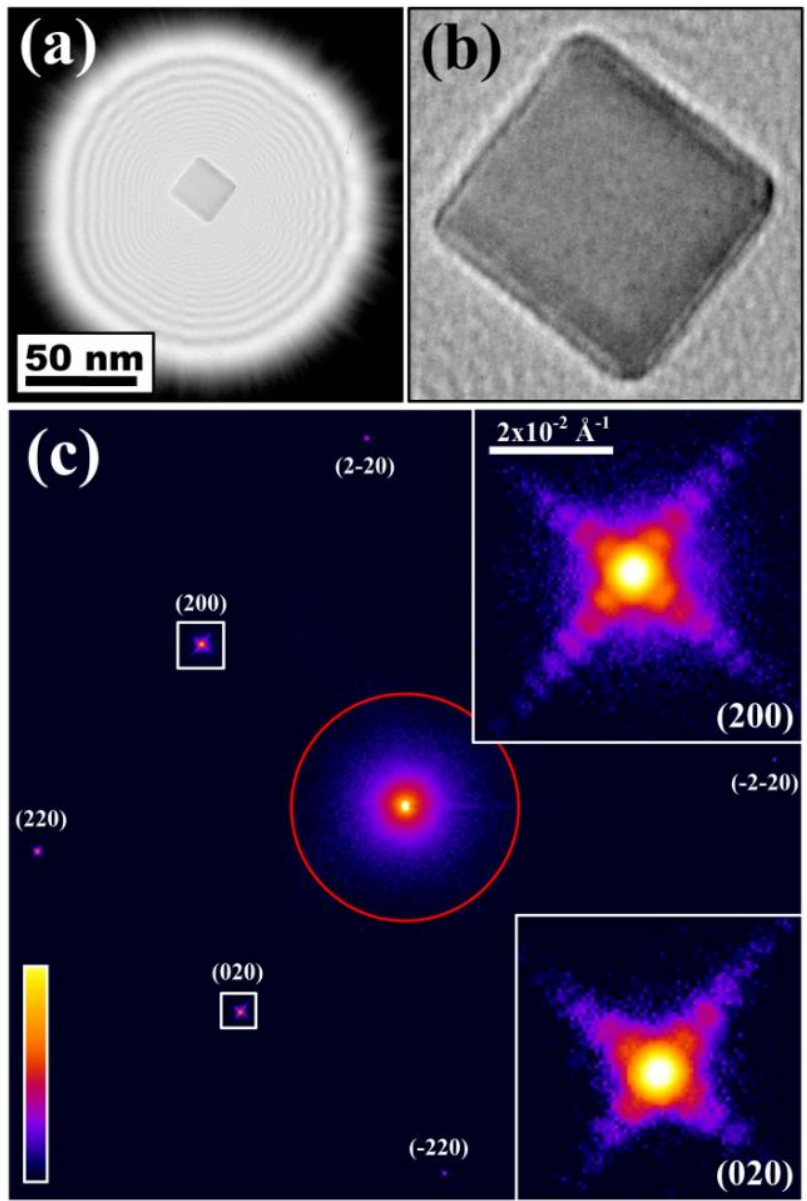

Fig. 1 (a) TEM image of the electron probe illuminating the sample (b) Enlarged image of the $\mathrm{MgO}$ nanoparticle shows faceted structure at corners and edges. (c) Electron diffraction pattern nanoparticle recovered from intensity around recorded on a Fuji imaging plate near the [001] zone axis orientation. Red line borders the area where scattering from amorphous substrate is localized. Insets, upper and bottom right are enlarged views of (200) and (020) Bragg peaks correspondingly
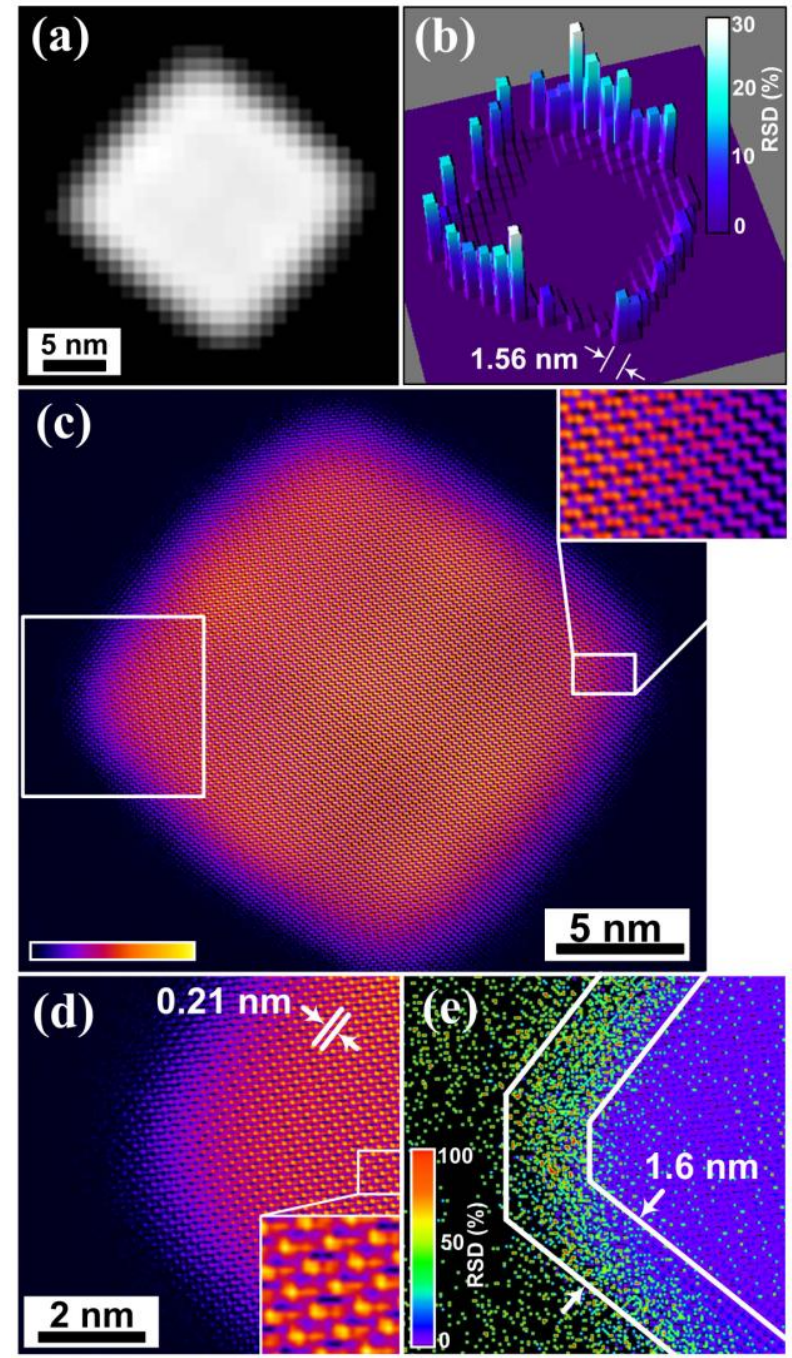

Fig. 2 (a) Image of the shape of $\mathrm{MgO}$
(200) Bragg peak. (b) Deviation map of solution showing the relative error of recovered shape which is higher within the one pixel $(1.56 \mathrm{~nm})$ at edges of the sample. (c) Amplitude of the reconstructed exit wave function with inset showing effect of slight tilt of the sample. (d) Enlarged view of the edge indicated by the square in (c). (e) Deviation map of the part of exit wave shown in (d). The region at edges and corners of the sample where spatial error of solution is high $(\sim 35 \%)$ is approximately $1.6 \mathrm{~nm}$. 